\title{
Estudio de un proceso de aprendizaje en ciencias experimentales a partir de la Teoría del Caos
}

\author{
Patricio R. Pacheco \\ Depto. de Física, Facultad de Ciencias Naturales, Matemática y del Medio Ambiente, Universidad Tecnológica \\ Metropolitana, Las Palmeras 3360, Santiago-Chile. (correo-e: patricio.pacheco@utem.cl)
}

Recibido Oct. 14, 2019; Aceptado Dic. 9, 2019; Versión final Ene. 31, 2020, Publicado Jun. 2020

\begin{abstract}
Resumen
El objetivo de esta investigación es demostrar que la transformación emocional, la conectividad y la entropía son básicas en un aprendizaje colaborativo. Las conductas pertenecientes al dominio de las emociones, la indagación, la persuasión y el uso de información desplegadas por equipos de alumnos en actividades experimentales de ciencias físicas son observadas y transformadas en series de tiempo. La teoría del caos es aplicada sobre estas series lo que da como resultado indicadores numéricos que ligan sus aprendizajes, rendimientos y calidad de nexos relacionales a la transformación de sus emociones. La modelación de las series de tiempo con las ecuaciones de Lorenz entrega la conectividad por equipo que junto a las emociones se emplean para caracterizar las dinámicas de aprendizaje. El cálculo de la entropía de correlación de las series de tiempo conduce, naturalmente, a la complejidad, a la sustentabilidad del proceso de aprendizaje revelando su conexión con los ecosistemas.
\end{abstract}

Palabras clave: series de tiempo; coeficientes de Lyapunov; conectividad; entropía

\section{Study of a learning process in experimental sciences by the Chaos Theory}

\begin{abstract}
The objective of this research is to demonstrate that emotional transformation, connectivity, and entropy is essential for collaborative learning. Behaviors belonging to the emotional domain, inquiry domain, persuasion domain, and information usage domain were observed in student teams performing physical science experiments. These behaviors were transformed into time series to which the Chaos theory was applied. The results obtained were numerical indicators that linked student learning, performance, and relationship link quality to the transformation of student emotions. The modeling of the time series with the Lorenz equations provided the connectivity per team. This was combined with the emotions to characterize learning dynamics. The correlation entropy of time series calculation results in complexity and in sustainability of the learning process revealing its connection with ecosystems.
\end{abstract}

Keywords: time series; Lyapunov coefficients; connectivity; entropy 


\section{INTRODUCCIÓN}

El caos posibilita la vida y la inteligencia ya que el cerebro es un producto no lineal de una evolución no lineal en un planeta no lineal (Briggs y Peat, 1999). El cerebro es un sistema inestable que sin embargo conduce y logra la formación de nuevos órdenes al igual que las actuaciones caóticas (Briggs y Peat, 1999). El cerebro ha evolucionado para volverse tan inestable que al menor estímulo o señal externa o interna (técnicamente una condición inicial), puede propiciar conductas que representan un saludable rompimiento con patrones históricos de conductas favoreciendo la emergencia de un orden innovador y creativo (Colom, 2001). Estos enunciados, fundados en observaciones, son la idea matriz de esta aproximación al análisis caótico de las observaciones de variables que tienen alta incidencia en el aprendizaje haciéndolo significativo.

Por otra parte investigaciones de diversos autores (Maturana, 2001; Goleman, 2005: Lavados, 2013; Pacheco, 2016; Gomes y Sprott, 2017; Pacheco, 2018; Blanco et al., 2019) señalan que las emociones cumplen un importante papel cognitivo: el conocimiento de la vida y el universo no es sólo intelectual, puesto que los matices más sutiles de él nos lo aporta la emotividad. Las emociones son la otra forma de saber sobre el mundo y sobre si mismos (Lavados, 2013). En el aprendizaje debería darse valor a hechos, personas y situaciones (que conforman la condición inicial contextualizada), según su influencia en la emocionalidad de los individuos y equipos. Esta asignación de valor se manifiesta, neurobiológicamente, en la selección atencional y perceptiva. En la selección de que se recuerda por la memoria de largo plazo, y en la percepción de que disposiciones y actitudes son "sentidas" como más apropiadas (Lavados, 2013; Gracia-Bafalluy y Escolano, 2014). Para investigar la influencia de las emociones en el aprendizaje se empleó un curso de Laboratorio de Mecánica Clásica (ver el esquema explicativo de la Figura 1) que permitió observar las conductas desplegadas por los equipos de alumnos en la consecución de los objetivo de la actividad. Las conductas, registradas a los equipos con instrumentos válidos y confiables, fueron transformadas en Series de Tiempo

El aprendizaje, además, es conectado y teje redes interactivas crecientes y de complejidad progresiva. Sigue el patrón de los sistemas presentes en la naturaleza que están sometidos a procesos irreversibles, es decir el sistema y sus alrededores no pueden regresar a su estado inicial. El fenómeno de la irreversibilidad según Prigogine tiene carácter constructivo (Prigogine, 1983), destacando el "papel creativo del tiempo", lo que, al menos a nivel macroscópico, supone una especie de antientropía: el universo del no equilibrio es un universo conexo. Frase que revela que entre conectividad e irreversibilidad existe un dialogo profundo. Una magnitud que da cuenta de sistemas que tienen esas características es la entropía. Esta es una variable fundamental que tiene diferentes definiciones y es medida en disímiles formas según los distintos campos en que se aplica (Haddad, 2017). Por ejemplo, en física clásica, la entropía de un sistema físico es proporcional a la cantidad de energía no disponible para hacer trabajo físico. La entropía es central en la Segunda Ley de la Termodinámica, que establece que en un sistema aislado cualquier actividad incrementa la entropía; en mecánica cuántica, von Neumann extendió la noción de entropía a sistemas cuánticos por medio de la matriz de densidad.

En teoría de probabilidades, la entropía de una variable azarosa mide la incerteza respecto del valor que debe ser asumido por la variable; en teoría de información, la entropía de compresión de un mensaje (un archivo computacional) cuantifica la cantidad de información que es llevada por el mensaje en términos de la mejor razón de compresión sin perdidas, en la teoría de sistemas dinámicos, la entropía cuantifica la complejidad exponencial de los sistemas o el flujo promedio de información por unidad de tiempo; en sociología, la entropía es el decaimiento natural de las estructuras ( tales como leyes, organización, y convenciones) de un sistema social; y en el sentido común, la entropía significa desorden o caos. Al decir que la entropía es una medida del desorden se da a entender que la más alta entropía es el más grande desorden, lo que aparece cuando es considerada como una medida de la no disponibilidad de la energía de un sistema para hacer trabajo; como el parámetro que representa el estado de desorden de un sistema atómico, iónico, o de nivel molecular; o una medida del desorden en el universo o simplemente como una medida del desorden de un sistema cualquiera (Ruelle, 2003; Liu et al., 2011). De aquí surgen las preguntas que orientan este trabajo. Al construir procedimientos experimentales que registran las conductas desplegadas por equipos de alumnos en proceso de aprendizaje y transformando estos registros en series de tiempo ¿se puede explicitar la relación entre aprendizaje, entropía y sustentabilidad del sistema de aprendizaje a partir de las series de tiempo? y ¿qué significado tiene esa relación?

Hay métodos de cálculo que, aplicados a Series de Tiempo dan los coeficientes de Lyapunov, el exponente de Hurst, la Dimensión de Correlación y la entropía de Kolmogorov (Kolmogorov, 1983). De allí surge la naturaleza caótica del proceso, su complejidad y pérdida de información, cantidades que admiten caracterizar, a partir de datos cuantitativos, la sustentabilidad (Wackernagel et al., 2002; Hui, 2006; Sayre, 2008) referida al aprendizaje. 
Desde la perspectiva de un modelo caotico, la condicion inicial juega un rol fundamental. Aplicada al estudio del aprendizaje (Pacheco, 2018), aparece contextualizada como un estímulo, susceptible de construcción, que apela a una muestra de individuos con experiencias similares. La narrativa a construir puede ser un experimento demostrativo breve, videos de corta duración, interacción entre el profesor y equipos de alumnos a través de preguntas, etc. La condición inicial contextualizada busca producir emociones positivas o negativas propiciando conductas de tipo adaptativas. La construcción usada se explica en la Figura. 1, a continuación:

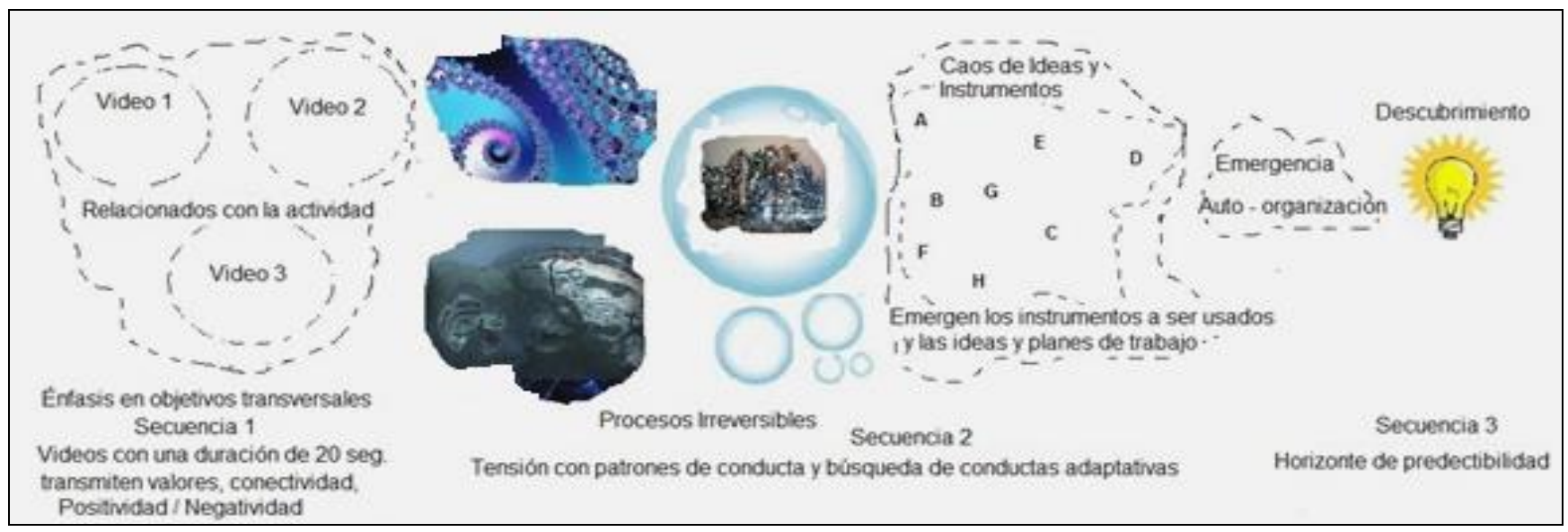

Fig. 1. Estructura de la narrativa visual, del orden de 300 seg., usada en cada actividad desarrollada del Laboratorio de Mecánica Clásica.

La secuencia 1 presenta tres o cuatro videos (cada uno de no más 10 a 15 segundos) disímiles o sin hilazón aparente con la actividad experimental que se realizara. Presentan objetivos transversales (respeto a los demás, mis actos tienen efectos sobre las personas y el medio ambiente, etc.). La secuencia 2 es una sesión de preguntas (alumnos - profesor) respecto a la conectividad de los eventos presenciados. Se busca estimular la curiosidad pero tensionando el ideal de mundo en el cual creemos vivir. El mundo real es de los procesos irreversibles, dominado por dinámicas no lineales, es decir el caos o de naturaleza fractal con bajo horizonte de predictibilidad. Se confronta lo aprendido y requerido con situaciones de alta incertidumbre produciéndose emociones de ira, negación, frustración, indiferencia, etc., que no resuelven el problema. Es conveniente disponer los instrumentos de medición, a emplear en la experiencia del laboratorio, en forma caótica, así a través de la auto organización y la emergencia los alumnos originan un orden que nos conduzca a una solución en la forma de descubrimiento. Las preguntas alumnos - profesor guían hacia la solución. La secuencia 3, final del video, expone para efectos comparativos, una disposición estándar de los instrumentos para alcanzar el objetivo de la actividad experimental de la sesión lectiva. Toda acción del ser humano conlleva incertidumbre. La experimentación, acorde como funciona nuestro cerebro, reduce la angustia, la frustración y el miedo.

La matriz de desempeño por equipo de alumnos se divide en intervalos de tiempo pequeños hasta completar la clase lectiva de 90 minutos. Se observan las conductas desplegadas por los alumnos en el proceso, para lo cual se tipifican 6 campos de conductas genéricas: Indagación (IND), Persuasión (PER), Positividad (POS), Negatividad (NEG), Información Interna (II) e Información Externa (IE). Cada campo contiene un conjunto de 13 conductas, asignándosele un rango numérico desde $-6 \mathrm{a}+6$, ver la Figura 2 de ejemplo para la Negatividad:

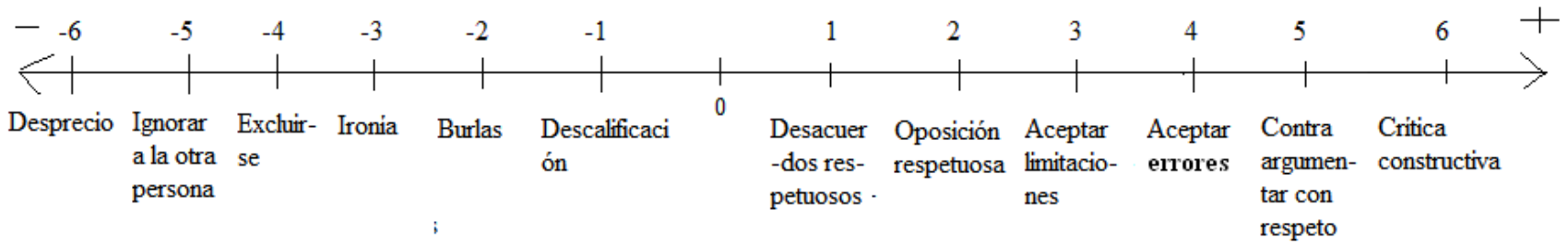

Fig. 2. Señala el dominio de doble polaridad y la escala de codificación, en su doble polaridad, de la Negatividad con sus trece conductas asociadas (Pacheco, 2016)

Se considera manifestada la conducta interactiva inter alumnos de un equipo para un tiempo de 5 segundos lo que da un total de 1080 filas de datos divididos a dos columnas. Una columna es la entrada del tiempo a intervalos de 5 segundos hasta completar 5400 segundos y la otra es la codificación numérica según la 
conducta observada desplegada por el equipo. En la construcción de las proporciones $X=I N D / P E R, Y=P O S$ / NEG, Z = II / IE se dividen los datos casillero por casillero, para el mismo tiempo, entre Indagación y Persuasión, Positividad y Negatividad, Información Interna e Información Externa, usando las 6 diferentes Tablas de Valores construidas. Esta jerarquización determina un total de 78 conductas a observar durante la sesión de 90 minutos.

\section{METODOLOGIA}

En el registro de las observaciones se utilizaron dos aulas acondicionadas como Laboratorio, ubicándose en cada una dos cámaras de video. Estas almacenan la información en discos duros externos. Las cámaras se posicionaron a una altura de $1.8 \mathrm{~m}$ en la pared y en forma tal que cubrieran completamente los equipos de alumnos los que a través de un documento firmado, autorizan la filmación. En un aula se ubicó el grupo experimental, con la condición inicial, y en la segunda sala se dispuso el grupo de control sin condición inicial. Al finalizar la sesión la información recopilada, con fecha, hora, actividad desarrollada y curso es guardada.

\section{Equipo para recopilar la información registrada en los videos}

Toda medición o instrumento de recolección de datos debe reunir dos requisitos esenciales: validez y confiabilidad (Wiersma, 1986; Gronlund, 1985, Pacheco, 2016 a). Aplicando el Test de Hipótesis o Docimasia a las Tablas de mediciones de las variables sometidas a observación se puede saber si el instrumento de medición está calibrado desde el punto de vista de la exactitud (Walpole et al., 2012). Para la extracción de la información de los videos se capacitó a observadores en las conductas definidas en esta investigación: Indagación, Persuasión, Positividad, Negatividad, Información Interna e Información Externa. (Ver ilustración en la Figura 2). ¿Es posible hacer una graduación más fina?, obviamente sí. No se realizó exploración específica de gestos faciales, por ejemplo, fruncir el ceño, abrir o cerrar los ojos (Ekman, 1993; Chansanchai, 2015; McDuff et al., 2016).

A los encargados del registro, constituido por 4 personas, se les entrega en forma independiente la misma escena, del orden de 10 minutos, de algunas de las actividades realizadas por los alumnos. Se codifican numéricamente según las conductas observadas para un mismo campo. Las Tablas de Codificación disponen de 1080 filas, separadas por intervalos de 5 segundos. Las Tablas generadas son analizadas por un psicólogo y un estadístico que estudian las convergencias y las divergencias entre los diferentes observadores: la clave es identificar las acciones que propician nexos al interior del equipo. Las escenas estudiadas se fueron ampliando gradualmente hasta los 90 minutos para efectos de análisis comparativo.

\section{Confiabilidad y validez de los instrumentos de medición}

La confiabilidad se verifica, para las tres variables $X, Y$ y Z a través del Coeficiente de Correlación Muestral de Pearson, Coeficiente Alfa de Cronbach (Cronbach, 1951), con Intervalo de Confianza al nivel de $\alpha=0.05$, Distribución de $\mathrm{t}$ - Student (Walpole et al., 2012). Para saber si el instrumento de medición está calibrado desde el punto de vista de la exactitud se aplicó la Prueba de Hipótesis definiéndose $\mathrm{H}_{0}=$ Hipótesis nula y $\mathrm{H}_{1}=$ Hipótesis alternativa. La Figura 3 representa la Distribución Normal de los indicadores t, para la variable Y. Permitió validar la hipótesis de instrumentos calibrados con exactitud al estar en la región de aceptación. Un procedimiento similar se utiliza para las variables X y Z (Pacheco, 2016).

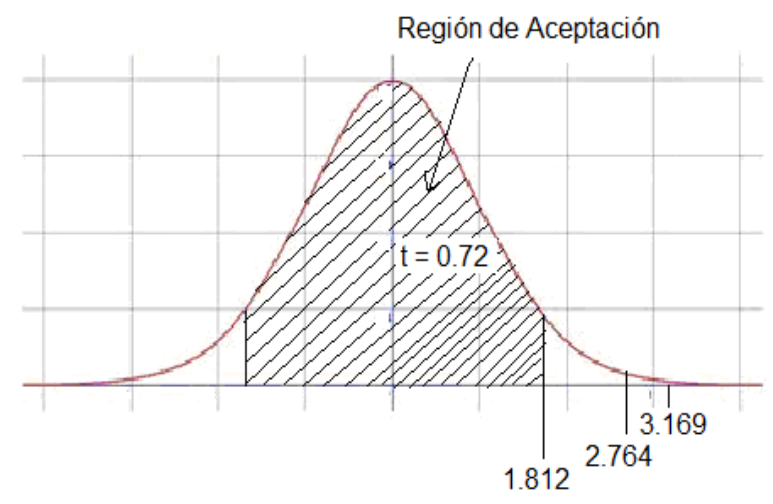

Fig. 3. Distribución Normal de los indicadores t. 
Series de tiempo y dinámicas de aprendizaje

Las proporciones $X(t)=I N D / P E R, \quad Y(t)=P O S / N E G, \quad Z(t)=I I / I E$ son series numéricas de 1080 datos que se denominan Series de Tiempo, vectores de1080 elementos (lo que permite hacer buenas estimaciones, lo ideal es que contengan por sobre los 5000 componentes para el análisis de estabilidad de los coeficientes de Lyapunov (Wolf et al., 1985). A partir $X(t), Y(t), Z(t)$ se obtienen las dinámicas de atractor débil $(r=$ parámetro de control $=17.5)$ y atractor medio $(r=20.5)$, que corresponden a rendimiento bajo y medio. El análisis caótico no aplica a estos atractores. En el caso del Atractor de Dinámica Caótica, $r=28.7$, (Grassberger y Procaccia, 1983), los gráficos de las Series de Tiempo y su evolución temporal (en el espacio de fases), se muestran en la Figura 4.
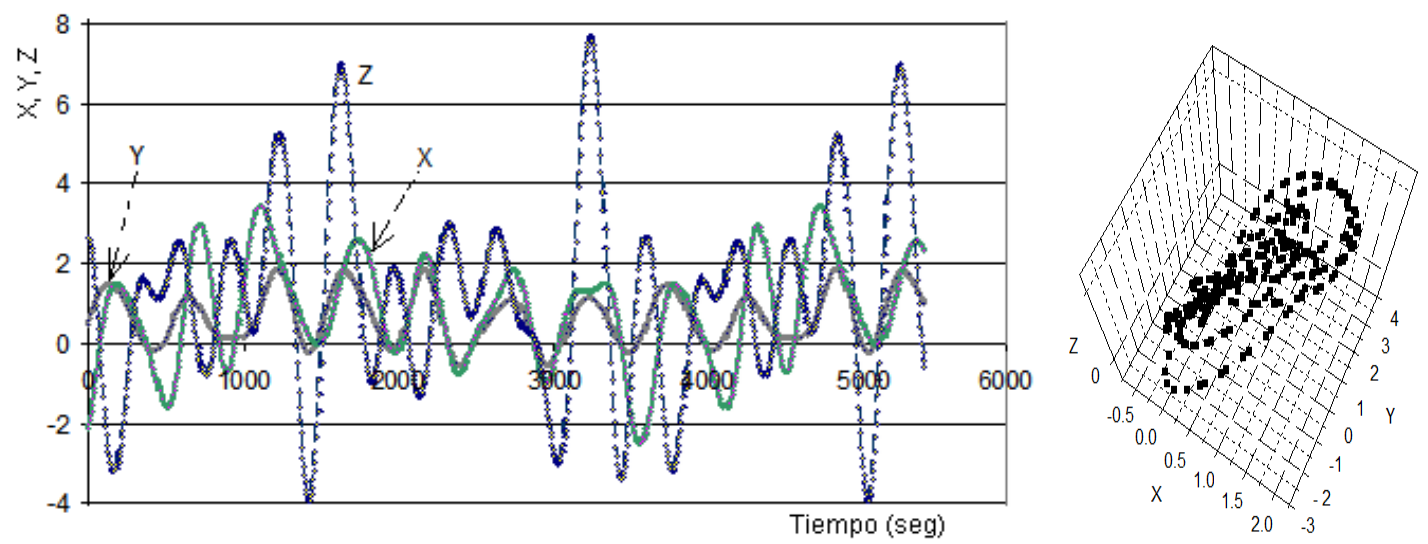

Fig. 4. Series de tiempo $X(t), Y(t), Z(t)$ de equipos de alumnos de alto rendimiento (dinámica caótica) y la configuración en el Espacio de Fases $X Y Z$ con $r=28.7$

Las Series de Tiempo posibilitan clasificar las dinámicas, según el aprendizaje significativo de los equipos que conforman el Grupo Experimental, en: Bajo, Medio y Alto. Se contrasta con el rendimiento de los Grupos de Control, de despliegue emocional en línea con un enfoque tradicional. Las correlaciones cruzadas por Grupo según influencia de la variable de las emociones $Y$ sobre la variable más racional $X$ del equipo (Pacheco, 2016), de dinámica caótica, alto rendimiento, se observa en la Tabla 1:

Tabla 1. Comparación entre correlación cruzada según Grupo de Control y Experimental

\begin{tabular}{|l|l|l|l|}
\hline Grupo & Control & Experimental & Comparación: Experimental / Control \\
\hline Correlación Cruzada & 0.3 & 0.5 & 1.7 \\
\hline
\end{tabular}

El grupo experimental favorece auto organización y emergencia con presencia equilibrada de POS / NEG en las relaciones. Al influir la parte emocional a la variable $\mathrm{X}$, la parte más racional del trabajo del equipo, en el orden $1.7 \sim 2$, conduce hacia mejores aprendizajes. Estos corolarios estimulan estudiar equipos con condición inicial e influencia de campo emocional (dinámica caótica en particular). Se aplica modelización al estudio de las Series de Tiempo a través del Software MatLab (Nakamura, 1997) ya sea por: ecuaciones de Lorenz (Lorenz, 1963; Sprott, 2006), Redes Neuronales o Autómatas Celulares (Wolfram, 1984). Los vectores X, Y y Z (Pacheco, 2016) se modelaron con las ecuaciones de Lorenz, pues tienen el parámetro de control (r) que se asocia a la conectividad, según el método numérico de Runge - Kutta de cuarto orden (Zill, 1988). El parámetro se relaciona con el rendimiento y el cuociente POS / NEG de las emociones en cada equipo (Pacheco, 2016), como presenta la Tabla 2:

Tabla 2. Muestra rendimientos según conectividad y cociente Positividad/Negatividad (POS/ NEG)

\begin{tabular}{|c|c|c|c|c|c|}
\hline & \multicolumn{3}{|c|}{ Cálculo de Conectividad según: } & & \\
\hline Rendimiento & Teoría & Centroide & Correlación & Promedio & POS / NEG \\
\hline Bajo (atractor débil) & 16.5 & 20.15 & 16 & 17.5 & 0.375 \\
\hline Medio (atractor medio) & 19.5 & 22.1 & 20 & 20.5 & 1.95 \\
\hline Alto (atractor caótico) & 31 & 26 & 29 & 28.7 & 5.25 \\
\hline
\end{tabular}


Los equipos de dinámica caótica tienen conectividad y POS / NEG alta, se sostienen en el tiempo y logran los objetivos de la actividad. Al observar la Tabla 2 se advierte un crecimiento de la conectividad en la medida que se aproxima a la dinámica caótica o compleja, como exhibe la Figura 5. ¿Qué significado tiene para el aprendizaje este comportamiento entrópico de la conectividad (que denominaremos conectividad entrópica)?, ¿es posible calcularlo?, y ¿cómo se relaciona con la complejidad del proceso de aprendizaje en estudio?

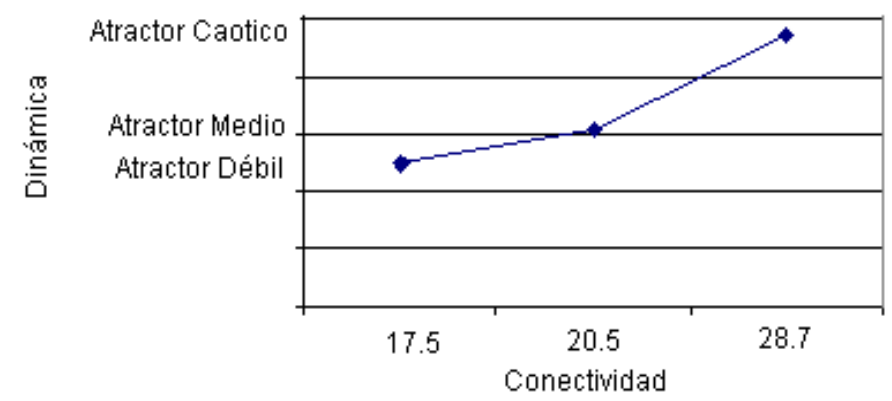

Fig. 5. Dinámicas de aprendizaje versus conectividad

Resultados de aplicar software de análisis caótico a las series de tiempo

Para el análisis de las Series de Tiempo se utilizó el Software CDA, Chaos Data Analyzer, Professional Version 2.2 (Sprott, 1998; Sprott, 2006). Notación: $\lambda$, exponente de Lyapunov (bits/unidades de tiempo) (Wolf et al.,1985); D: Dimensión de Incrustamiento (Sprott, 2006); n: es el número de intervalos de muestra sobre los cuales cada par de puntos es seguido antes de que un nuevo par sea seleccionado; A: es la exactitud relativa de los datos antes de que el ruido esperado comience a dominar; $\mathrm{H}$ : Exponente de Hurst está relacionado con la suavidad de la curva y con la dimensión fractal (Hurst et al.,1965), $0 \leq \mathrm{H} \leq 1$. Para $0.5 \leq \mathrm{H} \leq 1$ indica persistencia (el pasado tiende a persistir en el futuro); т es el tiempo de correlación, medido en segundos, calculado a partir del Software CDA y es, generalmente, del orden del recíproco del coeficiente Lyapunov; S: Entropía de correlación (Sprott, 2006) para cada variable (medida en bits / unidades de tiempo).

Tabla 3. Parámetros característicos de la Serie de Tiempo obtenidos por Teoría del Caos ( ${ }^{\star \star} D=3, n=1$ )

\begin{tabular}{|l|c|l|l|l|l|l|c|l|}
\hline Variable & $\lambda$, bit / seg. & $\mathrm{D}$ & $\mathrm{n}$ & $\mathrm{A}$ & $\mathrm{H}$ & Dimensión de Correlación & $\mathrm{T}$ & Entropía, bit / seg. \\
\hline $\mathrm{X}$ & $0.062 \pm 0.048$ & 3 & 1 & 0.0001 & 0.972 & $1.328 \pm 0.678^{* *}$ & 18.45 & $0.371^{* *}$ \\
\hline $\mathrm{Y}$ & $0.086 \pm 0.026$ & 3 & 1 & 0.0001 & 0.963 & $1.663 \pm 0.479^{* *}$ & 18.70 & $0.547^{* *}$ \\
\hline $\mathrm{Z}$ & $0.070 \pm 0.029$ & 3 & 1 & 0.0001 & 0.941 & $1.578 \pm 0.665^{* *}$ & 15.31 & $0.300^{* *}$ \\
\hline
\end{tabular}

En la Tabla 4 aparece el recíproco del exponente de Lyapunov, ie. el tiempo de Lyapunov, $t$ Lyapunov, la pérdida de información $(\mathrm{I}),<\Delta \mathrm{I}>\left(=<\mathrm{I}_{\text {NUEVA }}-\mathrm{I}_{\text {ANTIGUA }}>\right)$ y la dimensión fractal, D. Al considerar la relación entre la entropía de Kolmogorov y el parámetro característico del caos, el exponente de Lyapunov, $\lambda$, se desprende que éste es proporcional a la pérdida de información, $\langle\Delta I\rangle$ (Pacheco, 2018). Si $\lambda>0$ el movimiento es caótico, se pierde información, la predicción temporal es menos exacta y al ser la pérdida de información mayor no se puede hacer ninguna predicción fuera de ese horizonte de tiempo (Martínez y Vinagre, 2016). Los resultados de los cálculos se muestran en la Tabla 5.

$$
\langle\Delta \mathrm{I}\rangle_{\mathrm{i}} \log 2=-\lambda\left(\mathrm{i}_{0}(\mathrm{t})\right) \Rightarrow\langle\Delta \mathrm{I}\rangle_{\mathrm{i}}=\frac{-\lambda\left(\mathrm{i}_{0}(\mathrm{t})\right)}{\log 2}, \quad \mathrm{i}=\mathrm{X}, \mathrm{Y}, \mathrm{Z}
$$

Tabla 4. Perdida de Información, $\langle\Delta \mathrm{I}\rangle$, y Dimensión Fractal, D.

\begin{tabular}{|l|l|l|l|}
\hline Variable & \multicolumn{1}{|c|}{$\mathrm{t}$ Lyapunov } & \multicolumn{1}{|c|}{$<\mathrm{I}>$} & $\mathrm{D}=2-\mathrm{H}$ \\
\hline $\mathrm{X}$ & 16.13 & -0.206 & 1.0745 \\
\hline $\mathrm{Y}$ & 12 & -0.285 & 1.0862 \\
\hline $\mathrm{Z}$ & 14.3 & -0.232 & 1.1096 \\
\hline
\end{tabular}


La proximidad de los valores de $\mathrm{t}$ y $\mathrm{t}$ Lyapunov (comparando la Tabla 3 y la Tabla 4) para cada Serie de Tiempo da cuenta de la convergencia entre ambos métodos de aproximación. Un sistema con exponentes Lyapunov negativo no pierde, progresivamente, la información que tenía inicialmente y es, a partir de cierto tiempo, predecible (es la idea matriz del paradigma educativo actual). En el atractor caótico existe pérdida de información para $X(t), Y(t)$ y $Z(t)$, como muestra la tercera columna de la Tabla 4. Para $X(t)$ se obtiene $\langle\Delta \mathrm{l}\rangle_{\mathrm{x}}<0$ ya que $\lambda_{\mathrm{x}}$ $>0$, indicando que hay perdida de información, ¿cómo se entiende este resultado? y ¿qué nos sugiere? Se puede interpretar que el proceso desplegado por los equipos de alumnos de alto rendimiento, conducen a resultados ciertos (retroalimentación positiva) donde hay descubrimiento lo que hace el aprendizaje significativo pero con gran eficiencia para prescindir de información superflua, lo que en definitiva produce un decremento de la información hacia la efectivamente necesaria o relevante. Respecto de $Y(\mathrm{t})$ la pregunta a realizar, ¿es deseable que un sistema en proceso de aprendizaje se comporte de esta forma? Al asumir que hay más apertura en los equipos que trabajan colaborativamente, miran hacia adelante, sin seguir cargado a estereotipos y fijaciones emocionales (atractores de punto fijo). Las emociones exhiben complicaciones en su clasificación pues se trata de mecanismos neurobiológicos muy remotos y complejos (sus comienzos pueden indagarse en organismos unicelulares atraídos por variaciones de luz u oxigeno, que son capaces de "sentir"; feromonas que incitan conductas en insectos sociales, en las interacciones entre depredador- presa...) (Lavados, 2013; Gracia-Bafalluy y Escolano, 2014).

Así la dinámica caótica sobrelleva un aceptable rompimiento respecto de patrones de comportamiento que favorecen ciertas conductas repetitivas (Bijleveld et al., 2012) que frenan la creatividad demandada en contextos de alta conectividad - entre las personas, las personas con el ecosistema y el ecosistema consigo mismo - complejidad característica del mundo en que vivimos, que se resume "una mariposa bate sus alas en Hamburgo, Alemania, y un Tsunami afecta la costa de Constitución, Chile" (Brian et al.,1997). Los cálculos en Z (t), muestran pérdida de información, ¿cómo explicar este corolario? La razón entre las conductas exhibidas por el equipo en su proceso de búsqueda de información interna y externa al equipo, y que búsqueda prevalece. En procesos innovadores, es más lo que se descarta que lo conservado (probar estrategias nuevas lo que involucra, una disposición o un tono emocional diferente). Así $<\Delta I>x \quad \log 2<0$ es consecuente con lo observado.

El valor del exponente de Hurst indica que las formas relacionales generadas en los equipos de dinámica caótica propician persistencia (tenacidad) orientando el futuro. Desde esta perspectiva se conducen naturalmente a aprendizajes significativos, tal que la variable de las emociones $(Y)$ junto a la condición inicial contextualizada influencian a las Series de Tiempo de $X$ y de Z, ver Tabla 2 (el cociente POS/NEG es el mayor). ¿Cuál es el tiempo máximo que se puede proyectar, respecto de una actividad dada, el proceso de aprendizaje fundado en conectividad entrópica creciente en la Dinámica Caótica? Para el proceso colaborativo de aprendizaje investigado, de dinámica caótica, favorece la red de conectividad de un equipo de 4 personas, respecto de una actividad planteada como desafío o emprendimiento en un periodo lectivo que equivale a 90 minutos. La entropía de Kolmogorov (Pesin, 1977) es:

$$
\mathrm{S}_{\mathrm{K}}=-\sum \mathrm{p}_{\mathrm{i}} \log _{2} \mathrm{p}_{\mathrm{i}}=\sum_{\lambda>0} \lambda_{\mathrm{i}}=0.22 \text { bits/tiempo }
$$

Para el período lectivo, con los $\lambda_{i}$ valores máximos positivos de coeficientes de Lyapunov de la Serie de Tiempo. Este valor numérico indica que se pierden 0.2 bits de información por unidad de tiempo en los 90 minutos. Si bien existe perdida esta es relativamente baja lo que, indirectamente, indica que existe un porcentaje alto de retención durante la sesión. El tiempo de proyección para pérdida de la información del sistema, es:

$$
\text { Tiempo de Lyapunov }=\mathrm{t} \text { Lyapunov }=1 / \mathrm{S}_{\mathrm{K}}=1 / 0.22=4.54 \mathrm{seg} .=0.08 \text { minutos } \approx 0.1 \mathrm{~min} .
$$

Este horizonte temporal no depende del pasado histórico del proceso en función del cual se ha realizado la proyección. En los sistemas caóticos, es más favorable mirar a lo por venir, para acrecentar el nivel de confiabilidad de una proyección, que mirar hacia el pasado. Para valores menores en conectividad el proceso de aprendizaje su vuelve rígido o poco adaptativo lo que no propicia la autoorganización (Schuschny, 2001). Estas consideraciones conducen a la pregunta, ¿es posible construir un formalismo teórico para la complejidad y sustentabilidad de los procesos de aprendizaje que pueda ser verificado con los resultados obtenidos de la aplicación del Software CDA a las Series de Tiempo experimentales, surgidas del seguimiento observacional?. 


\section{Sustentabilidad}

Desde un punto de vista de una teoría físico-matemática para el aprendizaje colaborativo (López-Corona et al, 2015), es posible a partir de la energía libre de Hemholtz (Wheeler, 1998) obtener una definición-caracterización de la sustentabilidad de un proceso de aprendizaje. Es así que para todo sistema termodinámico abierto la entropía total, S, se puede escribir como la suma de la entropía interna del sistema, SiNTERNA, y la entropía externa, SeXterna, a él:

$$
\mathrm{S}=\mathrm{SINTERNA}_{\mathrm{N}} \mathrm{S}_{\text {EXTERNA }}=\mathrm{S}_{\mathrm{I}}+\mathrm{S}_{\mathrm{E}}
$$

La producción de entropía expresada como variación en el tiempo:

$$
\frac{d S}{d t}=\frac{d S_{1}}{d t}+\frac{S_{E}}{d t}
$$

La segunda ley de la Termodinámica establece que la producción interna de entropía debido a procesos irreversibles que ocurren dentro del sistema:

$$
\frac{d S_{1}}{d t}>0
$$

Para el caso especial donde las constricciones son constantes, la teoría termodinámica clásica irreversible establece que eventualmente el sistema alcanzará un estado termodinámico estacionario en el cual todas las variables microscópicas, incluyendo la entropía total, son estacionarias en el tiempo:

$$
\frac{\mathrm{dS}}{\mathrm{dt}}=0 \text {, entonces: } \frac{\mathrm{dS}_{\mathrm{I}}}{\mathrm{dt}}=-\frac{\mathrm{dS}_{\mathrm{E}}}{\mathrm{dt}} \Rightarrow \frac{\mathrm{d} \mathrm{S}_{\mathrm{E}}}{\mathrm{dt}}<0
$$

Un sistema así caracterizado, para mantenerse en un estado termodinámico estacionario estable, requiere un flujo negativo continuo de entropía hacia el interior del sistema. Esto puede visualizarse según la teoría de juegos. Dado dos equipos $A$ y $B$ y suponiendo que algunos jugadores de $A$ asumen que una estrategia, digamos $A$, es mejor que la otra sin importar como el otro equipo, B, juega. Claramente la población de $A$ que asumió esta postura decrecerá en el tiempo puesto que su adaptabilidad es más baja que la promedio y eventualmente desaparecerán. Luego toda la población estará con la estrategia B, así la adaptabilidad de B y el promedio de adaptabilidad son iguales y la producción de entropía será cero alcanzando el estado estacionario. Socialmente este proceso requiere un flujo negativo continuo de entropía el que no es sostenible (Por ejemplo, países que optan por una estrategia de desarrollo mala y un país por una buena, tarde o temprano todos querrán estar con la "buena" estrategia así el flujo negativo de entropía derivado del decrecimiento de los que optaron por la "mala" estrategia se revelará como, en última instancia, no sostenible).

La sustentabilidad comprendida como la capacidad de un sistema para alcanzar estados de mayor longevidad, se puede obtener minimizando la energía libre de Helmholtz (Wheeler, 1998):

$$
\mathrm{F}=\mathrm{U}-\mathrm{TS}
$$

Donde $\mathrm{T}$ es la temperatura del sistema asociada con la aleatoriedad interna y $\mathrm{U}$ es la energía interna asociada lla energía debida a interacciones. La energía libre de Helmholtz, se puede minimizar según dos formas. Una es minimizar $U$ y la otra es maximizar $S$. La mayoría de las veces la energía interna $U$ es una cantidad conservada o no puede ser controlada externamente, queda como única alternativa maximizar la entropía. Si la variación en el tiempo de la Entropía es mayor que cero:

$$
\frac{\mathrm{dS}}{\mathrm{dt}}>0
$$

La entropía crece y el sistema entra a una configuración más sustentable. Si la variación es menor que cero:

$$
\frac{d S}{d t}<0
$$


La entropía decrece y el sistema está en una configuración lejos de la sustentabilidad. Una producción de entropía positiva se logra cuando la adaptabilidad promedio es mayor que la local y esto se corresponde con juegos (o procesos) cooperativos, así se tiene un indicador cuantitativo para la medición de la sustentabilidad de un sistema. La interacción es parte integral del mundo real. Al incrementarse la entropía del sistema se vuelve más estable en el tiempo, más sustentable. Esto explica la relación entre información y entropía ya que la complejidad se mide a partir de la información una vez realizados todos los cálculos al sistema. La adaptabilidad es una medida de la capacidad de minimización de la energía libre de Helmholtz.

\section{Relación entre entropía, información y complejidad}

La conectividad alta (Pacheco, 2016), caso caótico del orden de 30, entre componentes de un equipo es condición de producción de entropía alta y viceversa: significa que un j - ésimo componente va estableciendo cada vez más conexiones con los otros componentes y, a su vez, éstos entre sí y con él. En la naturaleza, los sistemas abiertos "propician" un orden creciente en conectividad, mostrándose dinámicamente sustentables, con dinámica relacional evolutiva, incrementando su complejidad en el tiempo (Kaspar y Schuster, 1986). Si se supone una secuencia de caracteres, $X$, compuesta por valores (Serie de Tiempo) o símbolos, los que siguen una distribución de probabilidad $\mathrm{P}(\mathrm{x})$, se construye para la información, I (Shannon, 1948):

$$
\mathrm{I}=-\sum \mathrm{P}(\mathrm{x})_{\mathrm{i}} \log \mathrm{P}(\mathrm{x})_{\mathrm{i}}=\mathrm{S}
$$

Expresión que tiene la forma funcional de la entropía de las Ciencias Físicas. La complejidad según Gershenson (Gershenson y Fernández, 2012):

$$
\mathrm{C}=\mathrm{al}_{\text {out }}\left(1-\mathrm{I}_{\text {out }}\right)
$$

La letra "a" es una constante de normalización que hace adimensionada la cantidad C (un valor porcentual).Utilizando la entropía, S (López-Corona et al., 2015):

$$
\mathrm{C}=\mathrm{aS}_{\mathrm{obs}}\left(1-\mathrm{S}_{\mathrm{obs}}\right)=\mathrm{aS}(1-\mathrm{S})
$$

Si S MAX es la entropía máxima y da cuenta de la capacidad total de información del sistema entonces SoBs es la entropía observada y corresponde al contenido presente de información. La estructura formal construida conduce a una definición de la complejidad de un sistema, a través de su entropía. La entropía de Kolmogorov es la suma de coeficientes positivos de Lyapunov de las series de tiempo, la complejidad, en general, queda:

$$
\mathrm{C}(\mathrm{t})=\mathrm{aS}_{\mathrm{K}_{\mathrm{obs}}}\left(1-\mathrm{S}_{\mathrm{K}_{\mathrm{obs}}}\right)
$$

También se logra una buena aproximación a la Complejidad a partir de Series de Tiempo, usando función de correlación y matriz de correlación.

\section{Cálculo de complejidad}

La teoría caótica no aplica complejidad para atractor débil y atractor medio. En el caso del atractor extraño:

$$
\mathrm{S}_{\mathrm{K}}=\sum_{\lambda_{\mathrm{i}}>0} \lambda_{\mathrm{i}}=\lambda_{\mathrm{x}}+\lambda_{\mathrm{y}}+\lambda_{\mathrm{z}}=0.218 \approx 0.22 \frac{\text { bits }}{\text { tiempo }}>0
$$

De donde:

$$
\mathrm{S}_{\mathrm{K}} \Leftrightarrow \frac{\mathrm{dS}}{\mathrm{dt}} \text {, entonces: } \mathrm{S}_{\mathrm{K}} \Leftrightarrow \frac{\mathrm{dS}}{\mathrm{dt}}>0
$$

Desde el punto de vista de la aproximación de López - Corona (López-Corona et al., 2015) y usando el valor calculado de $S_{K}=0.218$ bits / tiempo $\approx 0.22$ bits / tiempo:

$$
\mathrm{C}(\mathrm{t})=\mathrm{aS}_{\mathrm{K}_{\mathrm{obs}}}\left(1-\mathrm{S}_{\mathrm{K}_{\mathrm{obs}}}\right)=\mathrm{a} 0.22(1-0.22) \approx 0.2>0
$$


El resultado de la complejidad es mayor que cero, característico de sistema complejo según la aproximación utilizada. De igual forma al usar las entropías de correlación para cada Serie de Tiempo se obtiene:

$$
C_{X}=0.23 \mathrm{a}, C_{Y}=0.26 \mathrm{a}, \mathrm{C}_{Z}=0.21 \mathrm{a}
$$

Y la suma total:

$$
\mathrm{C}_{\text {TOTAL }}=\mathrm{C}_{\mathrm{X}}+\mathrm{C}_{\mathrm{Y}}+\mathrm{C}_{Z} \approx 0.7 \mathrm{a}>0 .
$$

Esto señala que al crecer la entropía, el sistema de aprendizaje transita a configuraciones más complejas y sustentables, tal que la adaptabilidad media es mayor que la local dado que el carácter de la actividad en los equipos de alumnos se condice con procesos de aprendizaje colaborativos, generándose un indicador cuantitativo de la sustentabilidad del sistema. La entropía se relaciona con el parámetro de control del caso caótico con valor numérico del orden de 30 (Pacheco, 2016). Los miembros del equipo generan POS/NEG alta (mayores o iguales a: 2.5 (Bales, 1950); 4.3 (Schwartz y Weinberger, 1980), 5 (Gottman, 1994)).

\section{DISCUSION}

Este trabajo muestra que las conductas más favorables para un sistema de aprendizaje son bien descritas por dinámicas del tipo caótico. Entre las conductas, que se influencian, la emocional determina la conexión al interior de los equipos incidiendo en sus logros (Lavados, 2013). Son tales como compromiso, tenacidad, tolerancia a la frustración, colaboración respetuosa al interior del equipo, etc., "sentirse bien" con el desafío asumido, favoreciendo un aprendizaje que se descubre como significativo. Si bien el sentirse bien, un motor de búsqueda intrínseco de la emoción y la condición humana, estimula el desarrollo de la inventiva, la técnica y el consumo asociado, ha terminado adquiriendo una impronta de sobre exigencia extrema para el ecosistema del planeta (Mock 2013; Espirito-Santo et al., 2014; Moffitt et al., 2015; Paolo et al., 2015;Bar-On et al., 2018) al no sopesar su conectividad entrópica. La desatada exploración por generar más y perdurables momentos felices, sin mediar consecuencias, se esta revelando como no sostenible por el planeta, y el estudio realizado focaliza esta tensión y responsabilidad en nuestras conductas (Han, 2013). El desafío de los aprendizajes, conciliar aquello que nos singulariza como especie, la capacidad de transformar todo lo que nos rodea - incluyéndonos según nuestra reflexión y esperanza mediata con la incertidumbre o perdida en la información, lo que restringe severamente el horizonte de predictibilidad. En general, los sistemas caóticos se caracterizan por consumir considerable energía e información para poder mantener e incrementar su nivel de complejidad, a la vez que son muy sensibles a las condiciones iniciales (que pueden ser variaciones ambientales) (Asensio, 1997).

\section{CONCLUSIONES}

Según este estudio de primera aproximación las dinámicas de tipo caótico, logran aprendizajes significativos. Las Series de Tiempo exhiben dimensión fractal al calcular su exponente de Hurst, estando el rango entre $0.5<$ $\mathrm{H}<1$. Esto significa memoria de largo plazo contribuyendo a fortalecer la persistencia o perseverancia, al influenciarse entre ellas. La persistencia desborda las conductas asociadas a $X(t)$ y $Z(t)$, la parte más racional, asociándose a $Y(t)$ el dominio más emocional de conductas para el logro de los objetivos. Revelando naturaleza compleja, sensibilidad a condiciones iniciales y conectividad entre las variables con que se ha caracterizado el proceso de aprendizaje estudiado. Los rangos numéricos siguiendo los modelos teóricos analizados (López Corona et al., 2015; Sprott, 2006), indican las características que hacen sustentable el proceso de aprendizaje, demostrándose que dependen de: Conectividad creciente, cuociente de las emociones Positividad/Negatividad, Información e incerteza (Shannon, 1948; Kolmogorov, 1983) y Entropía. Parámetros empíricos, que permiten caracterizar la complejidad asociada al sistema de aprendizaje de la especie humana. Sistema que propiciará para sí aprendizaje significativo, rendimientos valorados en el paradigma de sociedad adscrito, auto organización, emergencia, diversidad y su sustentabilidad. Esta conclusión estimula, desde la perspectiva de la conectividad entrópica del aprendizaje, la reflexión sobre nuestra posición y actividad en el planeta que es, sin restar a los esfuerzos de largo plazo en viajes interplanetarios, el único lugar seguro (Lutz et al., 2004; Delp et al., 2016) que tenemos para vivir y compartir.

\section{AGRADECIMIENTOS:}

A la Universidad Tecnológica Metropolitana que a través de la Dirección de Investigación financió esta investigación y a su Dpto. de Física que colaboró con dependencias y equipamiento experimental.

\section{REFERENCIAS}


Asensio, J.M., Biología y educación, Ariel, Barcelona (1997).

Bales, R.F., Interaction process analysis: A Method for the Study of Small Groups, Addison - Wesley, Cambridge, Massachusetts, United States (1950)

Bar-On, Y.M., Phillips, R., y Milo, R., The biomass distribution on Earth, https://doi.org/10:1073/pnas.1711842115, Proceedings of the National Academy of Sciences, 115 (25), 6506-6511 (2018).

Bijleveld, E., Scheepers, D., y Ellemers, N., The Cortisol Response to Anticipated intergroup interactions Predicts SelfReported Prejuice, https://doi: 10.1371/journal.pone.0033681, PLoS One, 7(3), e33681 (2012).

Blanco, H., Peinado, J.E., Jurado, P.J., y Blanco, J.R., Invarianza Factorial del Cuestionario de Bienestar Psicológico de Ryff en Universitarios, de Acuerdo al Género, https://dx.doi.org/10.4067/S0718-50062019000300047, Formación Universitaria Vol. 12(3), 47-54 (2019)

Brian, A., Durlauf, S.N., y Lane, D.A., Introduction: Process and Emergence in the economy.The Economy as an Evolviing Complex System II, Addison - Wesley, Massachusets, United States (1997)

Briggs, J., y Peat, F.D., Las siete leyes del caos, Grijalbo, Barcelona (1999)

Chansanchai, A., Microsoft Project Oxford team creates tools that can recognize emotions in pictures (2015)

Colom, A. J., Teoría del caos y educación (acerca de la reconceptualización del saber educativo), Revista Española de Pedagogía, LIX, no 218, 5 - 24 (2001).

Cronbach, L. J., Coefficient alpha and the internal structure of tests, Psychometrika, 16(3), 297 - 334 (1951)

Delp, M. D., Charvat, J.M., Limoli, C.L., Globus, R.K., y Ghosh, P., Apollo Lunar Astronauts Show Higher Cardiovascular Disease Mortality: Possible Deep Space Radiation Effects on the Vascular Endothelium, https://doi: 10.1038/srep29901, Scientific Reports, 6, 29901 (2016)

Ekman, P., Facial Expression and Emotion, https://doi:10.1037/0003-066X.48.4.384, American Psychologist, 48 (4), 384-392 (1993)

Espirito-Santo, F. D. B., Gloor, M., y otros 19 autores, Size and frequency of natural forest disturbances and the Amazon forest carbon balance., https://doi:10.1038/ncomm s4434, Nature Communications, 5, 3434 (2014)

Gershenson, C., y Fernández, E., Complexity and Information: Measuring Emergente, Self - organization, and Homeostasis at Multiple Scales, https://doi: 10.1002/cplx.21424, arXiv, 1205-2026, v2[cs.TI] (2012)

Goleman, D., La Inteligencia Emocional, Kairós, Barcelona (2005)

Gomes, O., y Sprott, J.C., Sentiment-driven limit cycles and chaos, https://doi: 10.1007/s00191-017-0497-5, J Evol Econ., 27, 729-760 (2017)

Gottman, J., What predicts divorce? The relationship between marital processes and marital outcomes, Lawrence Erlbaum Associates, New Jersey (1994)

Gracia-Bafalluy, M., y Escolano, E., Aportaciones de la neurociencia al aprendizaje de las habilidades numéricas, Revista de Neurología, 58(2), 69 - 76 (2014)

Grassberger, P., y Procaccia, I., Characterization of Strange Attractors, https://doi.org/10.1103/Phys RevLett.50.346, Physical Review Letters, 50, 346-349. (1983)

Gronlund, N. E., Measurement and evaluation in teaching, Macmillan, Nueva York (1985)

Haddad, W., Thermodynamics: The Unique Universal Science, https://doi: 10.3390/e19110621, Entropy, 19(11), 621 (2017)

Han, Byung-Chul., La sociedad de la transparencia, Herder, Barcelona (2013).

Hui, C., Carrying capacity, population equilibrium, and environment's maximal load, https://dx.doi.org/10.1016/j. ecolmodel.2005. 07.001, Ecological Modelling, 192, 317-320. (2006).

Hurst, H. E., Black, R.P., y Simaika, Y.M., Long-term storage: an experimental study, Constable, London (1965)

Kaspar, F., y Schuster, H.G., Easily calculable measure for the complexity of spatiotemporal patterns, https://doi.org/10.1103/PhysRevA.36.842, Physical Review, A 36, 842 - 848 (1986)

Kolmogorov, A. N., Combinatorial foundations of information theory and the calculus of probabilities, Russian Mathematical Surveys, 38(4), $29-40$ (1983).

Lavados, J., El cerebro y la educación, Taurus, Santiago de Chile (2013).

Liu, Y., Liu, Ch., y Wang, D., Understanding Atmospheric Behaviour in Terms of Entropy: A Review of Applications of the Second Law of Thermodynamics to Meteorology, https://doi: 10.3390/cl3010211, Entropy, 13, $211-240$ (2011) 
López-Corona, O., Padilla, P., Huerta, A., Mustri - Trejo, D., Perez, K., Ruiz, A., Valdés, O., y Zamudio, F., Measuring social complexity and the emergence of cooperation from entropic principles, Physics-soc-ph.arXiv: 1502.05741v2[physics.Soc-ph] (19 de febrero del 2015)

Lorenz, E., Deterministic nonperiodic flow, https://doi: 10.1177/0309133308091948, Journal of atmospheric Sciences, 20, 130-141.(1963).

Lutz, W., Sanderson, W., y Scherbov, S., The End of the World Population Growth in the 21st Century: New Challenges for Human Capital Formation and Sustainable Development, Earthscan Publications Ltd, London (2004)

Martínez, J., y Vinagre, F., La entropía de Kolmogorov; su sentido físico y su aplicación al estudio de lechos fluidizados 2D. Depto. de Química Analítica e Ingeniería Química, Universidad de Alcalá, Alcalá de Henares. Madrid: Academia (2016).

Maturana, H., Emociones y Lenguaje en Educación y Política, Dolmen, Santiago de Chile (2001).

McDuff, D., Mahmoud, A., Mavadati, M., Amr, M., Turcot, J., y Kaliouby R., AFFDEX SDK: A Cross-Platform Real- Time Multi-Face Expression Recognition Toolkit, http://dx.doi.org/10.1145/2851581.2890247, Proceeding CHI EA 2016, Proceedings of the $2016 \mathrm{CHI}$ Conf. Extended Abstracts on Human Factors in Computing Systems, 3723 - 3726. (2016)

Mock, T., Climate Change Hill upset vital ocean chemical cycles, Journal Nature Climate Change, University of East Anglia press release 64 / 13. (9 de sept 2013).

Moffitt, S. E., Hill, T.M., Roopnarine, P.D., y Kennett, J.P., Response of seafloor ecosystems to abrupt global climate change, https://doi: 10.1073/pnas.1417130112, Proceedings of the National Academy of Sciences, 112 (15), 4684-4689 (2015).

Nakamura, S., Análisis Numérico y Visualización Gráfica con Matlab, Pearson, México D.F.(1997).

Pacheco, P., The Universe of Learning: Non-Equilibrium and Connectivity, In N. S. Columbus (Ed), Progress in Education (pp. 1 - 46), New York: Nova Publishing (2018).

Pacheco, P., Positividad y Negatividad emocional: influencia en los procesos de aprendizajes, https://dx.doi.org/ 10.4067/S0718-07052016000100012, Revista Estudios Pedagógicos, XLII, nº.1, 187-207 (2016)

Pacheco, P., Caos: conexión de aprendizajes, entropía, sustentabilidad y complejidad, EAE, .Madrid (2016 a)

Paolo, F.S., Fricker, H.A., y Padman, L., Volume loss from Antarctic ice shelves is acceleraying, https://doi:10.1126/science.aaa094 0, Science, 348(6232) 327-331 (2015).

Prigogine, I., ¿Tan sólo una ilusión?, Editorial Tusquets, Barcelona (1983)

Ruelle, D. P., Extending the definition of entropy to nonequilibrium steady states, https://doi.org/10.1073/ pnas.0630567100, Proceedings of the National Academy of Sciences, 100(6), $3054-3058$ (2003).

Sayre, N. F., The Genesis, History, and Limits of Carrying Capacity, https://dx.doi.org/10.1080/00045600701734356, Annals of the Assoc. of American Geo., 98(1), 120 - 134 (2008)

Schuschny, A., Auto organización en sistemas económicos, Buenos Aires: Biblioteca Digital de la Facultad de Ciencias Económicas - Universidad de Buenos Aires (2001).

Schwartz, G. E., y Weinberger, D.A., Patterns of emotional responses to affective situation: Relations among happiness, sadness, anger, fear, depression, and anxiety, Motivation and Emotion, 4(2), 175 - 191 (1980)

Shannon, C. A., Mathematical theory of communication, https://doi:10.1002/j.1538-7305.1948.tb01338.x, Bell System Technical Journal, 27, 379-423 \& $623-656$ (1948)

Sprott, J. C., Chaos and Time - Series Analysis, Oxford University Press, New York (2006).

Sprott, J.C., Chaos Data Analyzer, Professional Version 2.2 (1998).

Wackernagel, M., Schulz, N.B., y otros 9 autores, Tracking the ecological overshoot of the human economy, https://doi:10.1073/ pnas.142033699, Proceedings of the National Academy of Sciences, 99 (14), 9266 - 9271 (2002).

Walpole, R., Myers, R., Myers, S., y Ye, K., Probabilidad y Estadística para Ingeniería y Ciencias, Pearson, México, D.F. (2012).

Wheeler, L. P., Josiah Willard Gibbs. The History of a Great Mind, OxBow Press, Woodbridge, CT (1998).

Wiersma, W., Research methods in education: An introduction, Allyn and Bacon, Boston (1986).

Wolf, A., Swift, J.B., Swinney, H.L., y Vastano, J.A., Determining Lyapunov exponents from a time series, https://doi:10.1016/0167-2789(85)90011-9, Physica 16D, 285 - 317. North-Holland, Amsterdam (1985)

Wolfram, S., Cellular automata as models of complexity, https://doi:10.1038/311419a0, Nature, $311(5985)$, 419 - 424 (1984).

Zill, D., Ecuaciones Diferenciales con Aplicaciones, Grupo Editorial IberoAmérica S.A. de C.V, México, D.F. (1988) 